Moroccan J. of Pure and Appl. Anal. (MJPAA)

Volume 4(1), 2018, Pages 46-61

ISSN: Online 2351-8227 - Print 2605-6364

DOI 10.1515/mjpaa-2018-0006

\title{
Nonlocal eigenvalue problems with variable exponent
}

\author{
Elhoussine Azroul ${ }^{1, a}$ AND Mohammed Shimi ${ }^{1, b}$
}

ABSTRACT. We consider the nonlocal eigenvalue problem of the following form

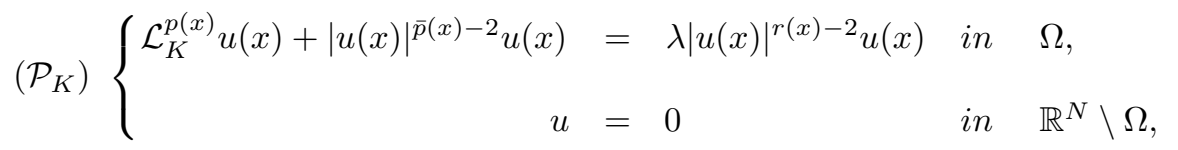

where $\Omega$ is a smooth open and bounded set in $\mathbb{R}^{N}(N \geqslant 3), \lambda>0$ is a real number, $K$ is a suitable kernel and $p, r$ are two bounded continuous functions on $\bar{\Omega}$. The main result of this paper establishes that any $\lambda>0$ sufficiently small is an eigenvalue of the above nonhomogeneous nonlocal problem. The proof relies on some variational arguments based on Ekeland's variational principle.

2010 Mathematics Subject Classification. Primary: 35R11, 35P30, 35J20, 35S05.

Key words and phrases. Generalized fractional Sobolev spaces with variable exponent, Nonlocal and pseudodifferential operators, eigenvalue problem, Ekeland's variational principle.

\section{Introduction}

We begin this work by remembering the definition of the fractional Sobolev spaces with variable exponent, to this end we follow the work of Pezzo and Rossi in [6].

Let $\Omega$ be a smooth bounded open set in $\mathbb{R}^{N}$. We start by fixing $s \in(0,1)$ and let $p: \bar{\Omega} \times \bar{\Omega} \longrightarrow(1,+\infty)$ be a continuous function. We assume that

$$
1<p^{-}=\min _{(x, y) \in \bar{\Omega} \times \bar{\Omega}} p(x, y) \leqslant p(x, y) \leqslant p^{+}=\max _{(x, y) \in \bar{\Omega} \times \bar{\Omega}} p(x, y)<+\infty
$$

and

$$
p \text { is symmetric, that is, } p(x, y)=p(y, x) \text { for all }(x, y) \in \bar{\Omega} \times \bar{\Omega}
$$

Received February 6, 2019 - Accepted March 17, 2019.

(C)The Author(s) 2017. This article is published with open access by Sidi Mohamed Ben Abdallah University.

${ }^{1}$ Sidi Mohamed Ben Abdellah University, Faculty of Sciences Dhar Al Mahraz, Laboratory of Mathematical Analysis and Applications, Fez, Morocco.

${ }^{a}$ e-mail: elhoussine.azroul@gmail.com

${ }^{b}$ e-mail: mohammed.shimi2@usmba.ac.ma. 
Let denote by :

$$
\bar{p}(x)=p(x, x) \text { for all } x \in \bar{\Omega} .
$$

We define the fractional Sobolev space with variable exponent via the Gagliardo approach as follows,

$$
\begin{aligned}
W= & W^{s, p(x, y)}(\Omega) \\
& =\left\{u \in L^{\bar{p}(x)}(\Omega): \int_{\Omega \times \Omega} \frac{|u(x)-u(y)|^{p(x, y)}}{\lambda^{p(x, y)}|x-y|^{s p(x, y)+N}} d x d y<+\infty, \text { for some } \lambda>0\right\},
\end{aligned}
$$

where $L^{\bar{p}(x)}(\Omega)$ is the Lebesgue space with variable exponent, (see section 2).

The space $W^{s, p(x, y)}(\Omega)$ is a Banach space (see [13]) if it is endowed with the norm,

$$
\|u\|_{W^{s, p(x, y)}(\Omega)}=\|u\|_{W}=\|u\|_{L^{\bar{p}(x)}(\Omega)}+[u]_{s, p(x, y)},
$$

where $[\cdot]_{s, p(x, y)}$ is a Gagliardo seminorm with variable exponent, which is defined by

$$
[u]_{s, p(x, y)}=\inf \left\{\lambda>0: \int_{\Omega \times \Omega} \frac{|u(x)-u(y)|^{p(x, y)}}{\lambda^{p(x, y)}|x-y|^{s p(x, y)+N}} d x d y \leqslant 1\right\} .
$$

The space $\left(W,\|\cdot\|_{W}\right)$ is a separable and reflexive, see [2, Lemma 3.1$]$.

Let us consider the fractional $p(x)$-Laplacian operator given by

$$
\left(-\Delta_{p(x)}\right)^{s} u(x)=p . v . \int_{\Omega} \frac{|u(x)-u(y)|^{p(x, y)-2}(u(x)-u(y))}{|x-y|^{n+s p(x, y)}} d y \text { for all } x \in \Omega,
$$

where p.v. is a commonly used abbreviation in the principal value sense.

Note that the operator $\left(-\Delta_{p(x)}\right)^{s}$ is the fractional version of well known $p(x)$-Laplacian operator $\Delta_{p(x)} u(x)=$ $\operatorname{div}\left(|\nabla u(x)|^{p(x)-2} u(x)\right)$. On the other hand, we remark that in the constant exponent case it is known as the fractional $p$-Laplacian operator $\left(-\Delta_{p}\right)^{s}$. This nonlocal nonlinear operator is consistent, up to some normalization constant depending upon $N$ and $s$, with the linear fractional Laplacian $(-\Delta)^{s}$ in the case $p=2$. The interest for this last operator and more generally pseudo-differential operators, has constantly increased over the last few years, although such operators have been a classical topic of functional analysis since long ago. Nonlocal operators such as $(-\Delta)^{s}$ and its generalisation $\mathcal{L}_{K}$ (see for instance $[12,14,20,21,22]$ ) naturally arise in continuum mechanics, phase transition phenomena, population dynamics and game theory, as they are the typical outcome of stochastical stabilization of Lévy processes, see e.g. $[4,16,17]$. We refer the reader to [3, 7] and to the references included for a self-contained overview of the basic properties of fractional Sobolev spaces and fractional Laplacian operator.

Now, we introduce the nonlocal integro-differential operator of elliptic type $\mathcal{L}_{K}^{p(x)}$ which generalizes $\left(-\Delta_{p(x)}\right)^{s}$, for any fixed $s \in(0,1)$, as follows:

$$
\begin{aligned}
\mathcal{L}_{K}^{p(x)}(u(x)) & =p . v \cdot \int_{\mathbb{R}^{N}}|u(x)-u(y)|^{p(x, y)-2}(u(x)-u(y)) K(x, y) d y \quad \text { for all } x \in \mathbb{R}^{N} \\
& =\lim _{\varepsilon \rightarrow 0} \int_{\mathbb{R}^{N} \backslash B_{\varepsilon}(x)}|u(x)-u(y)|^{p(x, y)-2}(u(x)-u(y)) K(x, y) d y \quad \text { for all } x \in \mathbb{R}^{N},
\end{aligned}
$$

where $p . v$. is a commonly used abbreviation in the principal value sense, $p: \mathbb{R}^{N} \times \mathbb{R}^{N} \longrightarrow(1,+\infty)$ be a continuous bounded function satisfying $(1.1),(1.2)$ on $\mathbb{R}^{N} \times \mathbb{R}^{N}$, and

$$
p((x, y)-(z, z))=p(x, y) \text { for all }(x, y),(z, z) \in \mathbb{R}^{N} \times \mathbb{R}^{N} .
$$

The kernel $K: \mathbb{R}^{N} \times \mathbb{R}^{N} \longrightarrow(0,+\infty)$ is a measurable function with the following properties

$$
K(x, y)=K(y, x) \quad \text { for any }(x, y) \in \mathbb{R}^{N} \times \mathbb{R}^{N},
$$

there exists a positive constant $k_{0}>0$ such that

$$
K(x, y) \geqslant k_{0}|x-y|^{-(N+s p(x, y))} \quad \text { for all }(x, y) \in \mathbb{R}^{N} \times \mathbb{R}^{N} \text { and } x \neq y,
$$




$$
m K \in L^{1}\left(\mathbb{R}^{N} \times \mathbb{R}^{N}\right) \text {, where } m(x, y)=\min \left\{1,|x-y|^{p(x, y)}\right\} .
$$

A typical example for $K$ is given by the singular kernel $K(x, y)=|x-y|^{-(N+s p(x, y))}$. In this case $\mathcal{L}_{K}^{p(x)}=$ $\left(-\Delta_{p(x)}\right)^{s}$.

An other example for $K$ is given by the kernel

$$
K_{1}(x, y)=|x-y|^{-(N+s p(x, y))} a(x-y),
$$

where $a: \mathbb{R}^{N} \longrightarrow[1,+\infty)$ is a bounded function, that is, $a \in L^{\infty}\left(\mathbb{R}^{N}\right)$. It is easy to see that $K_{1}$ satisfying $(1.4)$, (1.5) and (1.6).

Due to the non-localness of the operator $\mathcal{L}_{K}^{p(x)}$, we introduce the general fractional Sobolev space with variable exponent as follows

$$
E=W^{K, p(x, y)}(\Omega)=\left\{\begin{array}{c}
: \mathbb{R}^{N} \longrightarrow \mathbb{R} \text { measurable, such that } u_{\mid \Omega} \in L^{\bar{p}(x)}(\Omega) \text { with } \\
\int_{Q} \frac{|u(x)-u(y)|^{p(x, y)}}{\lambda^{p(x, y)}} K(x, y) d x d y<+\infty, \text { for some } \lambda>0
\end{array}\right\},
$$

where $\Omega$ be an open bounded subset of $\mathbb{R}^{N}$ and $Q$ defined by

$$
Q:=\mathbb{R}^{N} \times \mathbb{R}^{N} \backslash(\mathcal{C} \Omega \times \mathcal{C} \Omega), \quad \text { with } \mathcal{C} \Omega=\mathbb{R}^{N} \backslash \Omega .
$$

The space $W^{K, p(x, y)}(\Omega)$ is a Banach space (see [1]) if it is endowed with the norm

$$
\|u\|_{W^{K, p(x, y)}(\Omega)}=\|u\|_{K, p(x, y)}=\|u\|_{L^{\bar{p}(x)}(\Omega)}+[u]_{K, p(x, y)},
$$

where, $[u]_{K, p(x, y)}=\inf \left\{\lambda>0: \int_{Q} \frac{|u(x)-u(y)|^{p(x, y)}}{\lambda^{p(x, y)}} K(x, y) d x d y \leqslant 1\right\}$.

The space $\left(E,\|\cdot\|_{E}\right)$ is separable and uniformly convex reflexive, see ([1]).

We remark that even in the model case in which $K=|x-y|^{-(N+s p(x, y))}$, the norms $\|\cdot\|_{K, p(x, y)}$ and $\|\cdot\|_{s, p(x, y)}$ are not the same because $\Omega \times \Omega$ is strictly contained in $Q$ this makes the fractional Sobolev space with variable exponent approach $\left(W^{s, p(x, y)}(\Omega)\right)$ not sufficient for studying the nonlocal problem. For this we use the generalized fractional Sobolev space with variable exponent approach $\left(W^{K, p(x, y)}(\Omega)\right)$ have been considered in [1], in order to correctly encode the Dirichlet boundary datum in the variational formulation.

In this paper, we are concerned with the study of the following nonlocal eigenvalue problem

$$
\left(\mathcal{P}_{K}\right)\left\{\begin{aligned}
\mathcal{L}_{K}^{p(x)} u(x)+|u(x)|^{\bar{p}(x)-2} u(x) & =\lambda|u(x)|^{r(x)-2} u(x) & \text { in } & \Omega, \\
u & =0 & \text { in } & \mathbb{R}^{N} \backslash \Omega,
\end{aligned}\right.
$$

where $\Omega$ is a smooth open and bounded set in $\mathbb{R}^{N}(N \geqslant 3), \lambda>0$ is a real number, $p: \mathbb{R}^{N} \times \mathbb{R}^{N} \longrightarrow(1,+\infty)$ is a continuous function satisfying (1.1) and (1.2). And $r: \bar{\Omega} \longrightarrow(1,+\infty)$ is a bounded continuous function such that

$$
1<r^{-}=\min _{x \in \bar{\Omega}} r(x) \leqslant r(x) \leqslant r^{+}=\max _{x \in \bar{\Omega}} r(x)<p^{-} \text {for all } x \in \bar{\Omega} .
$$

Our purpose is to establish the existence of a continuous family of eigenvalues for problem $\left(\mathcal{P}_{K}\right)$ in a neighborhood of the origin. More precisely, we show that there exists $\lambda^{*}$ such that any $\lambda \in\left(0, \lambda^{*}\right)$ is an eigenvalue of the above problem.

In the context of eigenvalue problems involving variable exponent represent a starting point in analyzing more complicated equations. A first contribution in this sense is the paper of X. L. Fan, Q. H. Zhang and D. Zhao [11] where the following eigenvalue problem has been considered, 


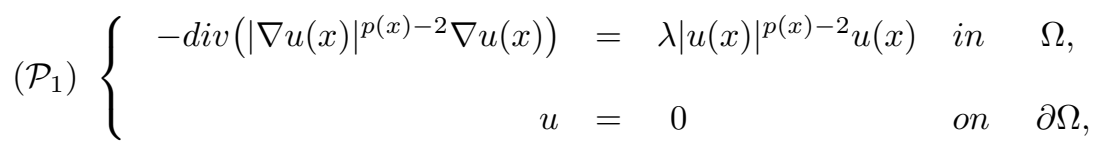

where $\Omega \subset \mathbb{R}^{N}(N \geqslant 3)$ is a bounded domain with smooth boundary $\partial \Omega$, the function $p: \bar{\Omega} \longrightarrow(1,+\infty)$ is continuous and $\lambda>0$ is a real number. The result obtained in [11] establishes the existence of infinitely many eigenvalues for problem $\left(\mathcal{P}_{1}\right)$ by using an argument based on the Ljusternik-Schnirelmann critical point theory. Denoting by $\Lambda$ the set of all nonnegative eigenvalues, the authors showed that sup $\Lambda=+\infty$ and they pointed out that only under special conditions, which are somehow connected with a kind of monotony of the function $p(x)$, we have inf $\Lambda>0$ (this is in contrast with the case when $p(x)$ is a constant; then, we always have inf $\Lambda>0$ ).

Going further, another eigenvalue problem involving variable exponent growth conditions intensively studied is the following

$$
\left(\mathcal{P}_{2}\right)\left\{\begin{aligned}
-\operatorname{div}\left(|\nabla u(x)|^{p(x)-2} \nabla u(x)\right) & =\lambda|u(x)|^{q(x)-2} u(x) & & \text { in } & \Omega, \\
u & =0 & & \text { on } & \partial \Omega,
\end{aligned}\right.
$$

where $p, q: \bar{\Omega} \longrightarrow(1,+\infty)$ are two continuous functions and $\lambda>0$ is a real number.

Note that when $p(x) \neq q(x)$, the competition between the growth rates involved in problem $\left(\mathcal{P}_{2}\right)$ is essential in describing the set of eigenvalues of this problem and we cite the following:

- In the case when $\min _{x \in \bar{\Omega}} q(x)<\min _{x \in \bar{\Omega}} p(x)$ and $q(x)$ has a subcritical growth, M. Mihăilescu and V. Rădulescu [19] used Ekeland's variational principle in order to prove the existence of a continuous family of eigenvalues which lies in a neighborhood of the origin. This result was later extended by X. Fan in [8].

- In the case when $\max _{x \in \bar{\Omega}} p(x)<\min _{x \in \bar{\Omega}} q(x)$ and $q(x)$ has a subcritical growth, a mountain pass argument, similar with that used by Fan and Zhang [9], can be applied in order to show that any $\lambda>0$ is an eigenvalue of problem $\left(\mathcal{P}_{2}\right)$.

- Finally, in the case when $\max _{x \in \bar{\Omega}} q(x)<\min _{x \in \bar{\Omega}} p(x)$ it can be proved that the energetic functional which can be associated with the eigenvalue problem has a nontrivial minimum for any positive $\lambda$ large enough (see, [9]). Clearly, in this case, the result of M. Mihăilescu and V. Rădulescu [19] can be also applied. Consequently, in this situation there exist two positive constants $\lambda^{*}$ and $\lambda^{* *}$ such that any $\lambda \in\left(0, \lambda^{*}\right) \cup\left(\lambda^{* *},+\infty\right)$ is an eigenvalue of the problem.

In an appropriate context we also point out the study of the eigenvalue problem,

$$
\left(\mathcal{P}_{3}\right)\left\{\begin{aligned}
-\operatorname{div}\left(\left(\left.\nabla u(x)\right|^{p_{1}(x)-2}+|\nabla u(x)|^{p_{2}(x)-2}\right) \nabla u(x)\right) & =\lambda|u(x)|^{q(x)-2} u(x) \text { in } \Omega, \\
u & =0 \text { on } \partial \Omega,
\end{aligned}\right.
$$

where $p_{1}, p_{2}, q: \bar{\Omega} \longrightarrow(1,+\infty)$ are continuous functions satisfying

$$
1<p_{2}(x)<\min _{x \in \bar{\Omega}} q(x) \leqslant \max _{x \in \bar{\Omega}} q(x)<p_{1}(x)<N \text { for all } x \in \bar{\Omega}
$$

and

$$
\max _{x \in \bar{\Omega}} q(x)<\frac{N p_{2}(x)}{N-p_{2(x)}} \text { for all } x \in \bar{\Omega} .
$$

For this problem M. Mihăilescu and V. Rădulescu [18] have proved the existence of two positive constants $\lambda_{0}$ and $\lambda_{1}$ with $\lambda_{0} \leqslant \lambda_{1}$ such that any $\lambda \in\left(\lambda_{1},+\infty\right)$ is an eigenvalue of problem $\left(\mathcal{P}_{3}\right)$ while any $\lambda \in\left(0, \lambda_{0}\right)$ is not an eigenvalue of problem $\left(\mathcal{P}_{3}\right)$.

One typical feature of problem $\left(\mathcal{P}_{K}\right)$ is the nonlocality, in the sense that the value of $\mathcal{L}_{K}^{p(x)}(u(x))$ at any point $x \in \Omega$ depends not only on the values of $u$ on $\Omega$, but actually on the entire space $\mathbb{R}^{N}$.

This paper is organized as follows. In section 2, we briefly review the definitions and collect some preliminary results for the Lebesgue spaces with variable exponent $L^{q(x)}$ and the generalized fractional Sobolev spaces with 
variable exponent $W^{K, p(x, y)}$. In section 3 , we introduce some important lemmas which show that the functional $\mathcal{J}_{\lambda}$ (see section 3) satisfies the geometrical conditions of the mountain pass theorem. Finally, using Ekeland's variational principle we prove that the problem $\left(\mathcal{P}_{K}\right)$ has a continuous spectrum which concentrates around the origin.

\section{Some preliminary results}

In this section, we recall some necessary properties of variable exponent spaces. For more details we refer the reader to $[5,10,15]$, and the references therein.

Consider the set

$$
C_{+}(\bar{\Omega})=\{q \in C(\bar{\Omega}): q(x)>1, \text { for all } x \in \bar{\Omega}\} .
$$

For all $q \in C_{+}(\bar{\Omega})$, we set

$$
q^{+}=\sup _{x \in \bar{\Omega}} q(x) \text { and } q^{-}=\inf _{x \in \bar{\Omega}} q(x) .
$$

Such that

$$
1<q^{-} \leqslant q(x) \leqslant q^{+}<+\infty
$$

For any $q \in C_{+}(\bar{\Omega})$, we define the variable exponent Lebesgue space as

$$
L^{q(x)}(\Omega)=\left\{u: \Omega \longrightarrow \mathbb{R} \text { measurable }: \int_{\Omega}|u(x)|^{q(x)} d x<+\infty\right\} .
$$

This vector space endowed with the Luxemburg norm, which is defined by

$$
\|u\|_{L^{q(x)}(\Omega)}=\inf \left\{\lambda>0: \int_{\Omega}\left|\frac{u(x)}{\lambda}\right|^{q(x)} d x \leqslant 1\right\}
$$

is a separable reflexive Banach space.

Let $\hat{q} \in C_{+}(\bar{\Omega})$ be the conjugate exponent of $q$, that is, $\frac{1}{q(x)}+\frac{1}{\hat{q}(x)}=1$. Then we have the following Hölder-type inequality

Lemma 2.1. (Hölder inequality). If $u \in L^{q(x)}(\Omega)$ and $v \in L^{\hat{q}(x)}(\Omega)$; then

$$
\left|\int_{\Omega} u v d x\right| \leqslant\left(\frac{1}{q^{-}}+\frac{1}{\hat{q}^{-}}\right)\|u\|_{L^{q(x)}(\Omega)}\|v\|_{L^{\hat{q}(x)}(\Omega)} \leqslant 2\|u\|_{L^{q(x)}(\Omega)}\|v\|_{L^{\hat{q}(x)}(\Omega)}
$$

A very important role in manipulating the generalized Lebesgue spaces with variable exponent is played by the modular of the $L^{q(x)}(\Omega)$ space, which defined by

$$
\begin{aligned}
\rho_{q(.)}: L^{q(x)}(\Omega) & \longrightarrow \mathbb{R} \\
u \longrightarrow \rho_{q(.)}(u)=\int_{\Omega}|u(x)|^{q(x)} d x &
\end{aligned}
$$

Proposition 2.1. Let $u \in L^{q(x)}(\Omega)$, then we have,

(1) $\|u\|_{L^{q(x)}(\Omega)}<1($ resp. $=1,>1) \Leftrightarrow \rho_{q(.)}(u)<1$ (resp. $\left.=1,>1\right)$,

(2) $\|u\|_{L^{q(x)}(\Omega)}<1 \Rightarrow\|u\|_{L^{q(x)}(\Omega)}^{q+} \leqslant \rho_{q(.)}(u) \leqslant\|u\|_{L^{q(x)}(\Omega)}^{q-}$,

(3) $\|u\|_{L^{q(x)}(\Omega)}>1 \Rightarrow\|u\|_{L^{q(x)}(\Omega)}^{q-} \leqslant \rho_{q(.)}(u) \leqslant\|u\|_{L^{q(x)}(\Omega)}^{q+}$.

Proposition 2.2. If $u, u_{k} \in L^{q(x)}(\Omega)$ and $k \in \mathbb{N}$, then the following assertions are equivalent:

(1) $\lim _{k \rightarrow+\infty}\left\|u_{k}-u\right\|_{L^{q(x)}(\Omega)}=0$,

(2) $\lim _{k \rightarrow+\infty} \rho_{q(.)}\left(u_{k}-u\right)=0$.

In [1], the authors have proved the following basic theorem. 
Theorem 2.1. Let $\Omega$ be a smooth bounded domain in $\mathbb{R}^{N}$ and let $s \in(0,1)$. Let $p: \mathbb{R}^{N} \times \mathbb{R}^{N} \longrightarrow(1,+\infty)$ be a continuous variable exponent with $s p(x, y)<N$ for all $(x, y) \in \mathbb{R}^{N} \times \mathbb{R}^{N}$. Let (1.1) and (1.2) be satisfied. Let $r: \bar{\Omega} \longrightarrow(1,+\infty)$ be a continuous bounded variable exponent such that,

$$
1<r(x)<p_{s}^{*}(x)=\frac{N \bar{p}(x)}{N-s \bar{p}(x)} \text { for all } x \in \bar{\Omega} .
$$

Suppose that $K: \mathbb{R}^{N} \times \mathbb{R}^{N} \longrightarrow(0,+\infty)$ is a measurable function satisfying (1.4), (1.5) and (1.6). Then

(1) There exists a positive constant $C=C(N, p, r, s, \Omega)>0$, such that for any $u \in W^{K, p(x, y)}(\Omega)$, one has

$$
\|u\|_{L^{r(x)}(\Omega)} \leqslant C\|u\|_{W^{s, p(x, y)}(\Omega)} \leqslant C \max \left\{1, \tilde{k}_{0}\right\}\|u\|_{K, p(x, y)}=\tilde{C}\|u\|_{K, p(x, y)},
$$

(2) there exists a positive constant $C_{0}=C_{0}\left(N, p, s, \tilde{k}_{0}, \Omega\right)>0$, such that

$$
[u]_{K, p(x, y)} \leqslant\|u\|_{K, p(x, y)} \leqslant C_{0}[u]_{K, p(x, y)} .
$$

For any $u \in W^{K, p(x, y)}(\Omega)$, we define the functional

$$
\rho_{K, p(., .)}(u)=\int_{Q}|u(x)-u(y)|^{p(x, y)} K(x, y) d x d y+\int_{\Omega}|u(x)|^{\bar{p}(x)} d x
$$

It is easy to see that $\rho_{K, p(. .)}$ is a convex modular on $W^{K, p(x, y)}(\Omega)$. The norm associated with $\rho_{K, p(. .)}$ is given by

$$
\|u\|_{\rho_{K, p(., .)}}=\inf \left\{\lambda>0: \rho_{K, p(., .)}\left(\frac{u}{\lambda}\right) \leqslant 1\right\} .
$$

Remark 2.1. $\rho_{K, p(.,)}$ also check the results of Propositions 2.1 and 2.2 .

Using the same argument as in [5, Theorem 2.17], we prove that $\|\cdot\|_{\rho_{K, p(., .)}}$ is a norm on $W^{K, p(x, y)}(\Omega)$, which is equivalent to the norm $\|\cdot\|_{K, p(x, y)}$.

We also define the closed linear subspace of $W^{K, p(x, y)}(\Omega)$ by

$$
E_{0}=W_{0}^{K, p(x, y)}(\Omega)=\left\{u \in W^{K, p(x, y)}(\Omega): u(x)=0 \text { a.e. } \in \mathbb{R}^{N} \backslash \Omega\right\} .
$$

\section{Remark 2.2.}

(1) The assertion $(i)$ in Theorem 2.1 implies that $W^{K, p(x, y)}(\Omega)$ and $W_{0}^{K, p(x, y)}(\Omega)$ are continuously embedded in $L^{r(x)}(\Omega)$, where $1<r(x)<p_{s}^{*}(x)$ for all $x \in \bar{\Omega}$.

(2) As a consequence of in Theorem 2.1-(ii), [.] $]_{K, p(x, y)}$ is an equivalent norm of $\|u\|_{K, p(x, y)}$ on $W_{0}^{K, p(x, y)}(\Omega)$.

(3) $\left(W_{0}^{K, p(x, y)}(\Omega),[\cdot]_{K, p(x, y)}\right)$ is a separable, reflexive, and uniformly convex Banach space (see [1, Lemma $3.5])$.

On the other hand, for any $u \in W_{0}^{K, p(x, y)}(\Omega)$, we define the functional

$$
\rho_{K, p(.,)}^{o}(u)=\int_{Q}|u(x)-u(y)|^{p(x, y)} K(x, y) d x d y .
$$

$\rho_{K, p(., .)}^{o}$ is a convex modular on $W_{0}^{K, p(x, y)}(\Omega)$. The norm associated with $\rho_{K, p(., .)}^{o}$ is given by

$$
\|u\|_{\rho_{K, p(., .)}^{o}}=[u]_{K, p(x, y)}=\inf \left\{\lambda>0: \rho_{K, p(., .)}^{o}\left(\frac{u}{\lambda}\right) \leqslant 1\right\}=\|u\|_{E_{0}} .
$$

\section{Remark 2.3.}

(1) $\rho_{K, p(., .)}^{o}$ also check the results of Proposition 2.2. 
(2) The modular $\rho_{K, p(., .)}^{o}$ does not satisfy the triangle inequality, that is,

$$
\rho_{K, p(., .)}^{o}(u+v) \leqslant \rho_{K, p(., .)}^{o}(u)+\rho_{K, p(., .)}^{o}(v)
$$

However, there is a substitute that is sometimes useful.

$$
\rho_{K, p(., .)}^{o}(u+v) \leqslant 2^{p^{+}-1}\left(\rho_{K, p(., .)}^{o}(u)+\rho_{K, p(., .)}^{o}(v)\right) .
$$

We will refer to this as the modular triangle inequality.

We could also get the following properties:

Lemma 2.2. ([1]). Let $p: \mathbb{R}^{N} \times \mathbb{R}^{N} \longrightarrow(1,+\infty)$, be a continuous variable exponent and let $K: \mathbb{R}^{N} \times \mathbb{R}^{N} \longrightarrow$ $(0,+\infty)$ is a measurable function satisfy (1.4) and (1.5). Then For any $u \in W_{0}^{K, p(x, y)}$, we have

(1) $1 \leqslant[u]_{K, p(x, y)} \Rightarrow[u]_{K, p(x, y)}^{p^{-}} \leqslant \rho_{K, p(., .)}^{o}(u) \leqslant[u]_{K, p(x, y)}^{p^{+}}$,

(2) $[u]_{K, p(x, y)} \leqslant 1 \Rightarrow[u]_{K, p(x, y)}^{p^{+}} \leqslant \rho_{K, p(., .)}^{o}(u) \leqslant[u]_{K, p(x, y)}^{p^{-}}$.

Lemma 2.3. ([1]) Let (1.1), (1.2) and (1.3) be satisfied. Then the space $C_{0}^{\infty}\left(\mathbb{R}^{N}\right)$ of smooth functions with compact support is dense in $W^{K, p(x, y)}(\Omega)$.

Let (1.1) and (1.2), be satisfied and let $K: \mathbb{R}^{N} \times \mathbb{R}^{N} \longrightarrow(0,+\infty)$ is a measurable function satisfy (1.4), (1.5) and (1.6). Then

$$
\begin{aligned}
\mathcal{L}_{K}^{p(x)}: E_{0} & \longrightarrow E_{0}^{*} \\
u & \longmapsto \mathcal{L}_{K}^{p(x)}(u): E_{0} \longrightarrow \mathbb{R} \\
\varphi & \longmapsto<\mathcal{L}_{K}^{p(x)}(u), \varphi>
\end{aligned}
$$

such that

$$
<\mathcal{L}_{K}^{p(x)}(u), \varphi>=\int_{\mathbb{R}^{2 N}}|u(x)-u(y)|^{p(x, y)-2}(u(x)-u(y))(\varphi(x)-\varphi(y)) K(x, y) d x d y .
$$

Where $E_{0}^{*}=\left(W_{0}^{K, p(x, y)}(\Omega)\right)^{*}$ is the dual space of $E=W_{0}^{K, p(x, y)}(\Omega)$.

In the following Lemma, we introduce fundamental properties of the operator $\mathcal{L}_{K}^{p(x)}$.

Lemma 2.4. ([1]) Suppose that (1.1) and (1.2) be satisfied and let $K: \mathbb{R}^{N} \times \mathbb{R}^{N} \longrightarrow(0,+\infty)$ be a measurable function which satisfies (1.4), (1.5) and (1.6). Then, The following assertions hold:

(1) $\mathcal{L}_{K}^{p(x)}$ is well defined and bounded,

(2) $\mathcal{L}_{K}^{p(x)}$ is a strictly monotone operator,

(3) $\mathcal{L}_{K}^{p(x)}$ is a mapping of type $\left(S_{+}\right)$, that is, if $u_{k} \rightarrow u$ in $E_{0}$ and lim $\sup <\mathcal{L}_{K}^{p(x)}(u)_{k}-\mathcal{L}_{K}^{p(x)}(u), u_{k}-u>\leqslant 0$, then $u_{k} \longrightarrow u$ in $W_{0}^{K, p(x, y)}$,

(4) $\mathcal{L}_{K}^{p(x)}$ is a homeomorphism,

(5) $\mathcal{L}_{K}^{p(x)}$ is coercive.

\section{Main results}

Definition 3.1. We say that $u \in E_{0}$ is a weak solution of problem $\left(\mathcal{P}_{K}\right)$, if for all $\varphi \in E_{0}$, we have

$$
\begin{aligned}
& \int_{Q}|u(x)-u(y)|^{p(x, y)-2}(u(x)-u(y))(\varphi(x)-\varphi(y)) K(x, y) d x d y \\
& +\int_{\Omega}|u(x)|^{\bar{p}(x)-2} u(x) \varphi(x) d x-\lambda \int_{\Omega}|u(x)|^{r(x)-2} u(x) \varphi(x) d x=0 .
\end{aligned}
$$


Moreover, we say that $\lambda$ is an eigenvalue of problem $\left(\mathcal{P}_{K}\right)$, if there exists $u \in E_{0} \backslash\{0\}$ which satisfies (3.1), i.e. $u$ is the corresponding eigenfunction to $\lambda$.

Let us consider the energy functional $\mathcal{J}_{\lambda}$ corresponding to problem $\left(\mathcal{P}_{K}\right)$, defined by, $\mathcal{J}_{\lambda}: W_{0}^{K, p(x, y)}(\Omega) \longrightarrow \mathbb{R}$

$$
\mathcal{J}_{\lambda}(u)=\int_{Q} \frac{1}{p(x, y)}|u(x)-u(y)|^{p(x, y)} K(x, y) d x d y+\int_{\Omega} \frac{1}{\bar{p}(x)}|u(x)|^{\bar{p}(x)} d x-\lambda \int_{\Omega} \frac{1}{r(x)}|u(x)|^{r(x)} d x,
$$

for any $\lambda>0$.

Now, we introduce some important lemmas that show that the functional $\mathcal{J}_{\lambda}$ satisfies the geometrical conditions of the mountain pass theorem which are necessary to establish the proof of the existence result.

Lemma 3.1. Let $\Omega$ be a smooth bounded domain in $\mathbb{R}^{N}$ and let $s \in(0,1)$. Let $p: \mathbb{R}^{N} \times \mathbb{R}^{N} \longrightarrow(1,+\infty)$ be a continuous variable exponent with $\operatorname{sp}(x, y)<N$ for all $(x, y) \in \mathbb{R}^{N} \times \mathbb{R}^{N}$. Let (1.1) and (1.2) be satisfied. Let $r: \bar{\Omega} \longrightarrow(1,+\infty)$ be a continuous bounded variable exponent satisfy $(1.7)$. Suppose that $K: \mathbb{R}^{N} \times \mathbb{R}^{N} \longrightarrow(0,+\infty)$ is a measurable function satisfying (1.4), (1.5) and (1.6). Then:

(1) $\mathcal{J}_{\lambda}$ is well defined.

(2) $\mathcal{J}_{\lambda} \in C^{1}\left(W_{0}^{K, p(x, y)}(\Omega), \mathbb{R}\right)$ and for all $u, \varphi \in W_{0}^{K, p(x, y)}(\Omega)$, its Gâteaux derivative is given by:

$$
\begin{aligned}
<\mathcal{J}_{\lambda}^{\prime}(u), \varphi>=\int_{Q}|u(x)-u(y)|^{p(x, y)-2}(u(x)-u(y))(\varphi(x)-\varphi(y)) K(x, y) d x d y \\
+\int_{\Omega}|u(x)|^{\bar{p}(x)-2} u(x) \varphi(x) d x-\lambda \int_{\Omega}|u(x)|^{r(x)-2} u(x) \varphi(x) d x
\end{aligned}
$$

Proof. (i)- Let $u \in W_{0}^{K, p(x, y)}(\Omega)$; then

$$
\begin{aligned}
\mathcal{J}_{\lambda}(u)= & \int_{Q} \frac{1}{p(x, y)}|u(x)-u(y)|^{p(x, y)} K(x, y) d x d y+\int_{\Omega} \frac{1}{\bar{p}(x)}|u(x)|^{\bar{p}(x)} d x \\
& \quad-\lambda \int_{\Omega} \frac{1}{r(x)}|u(x)|^{r(x)} d x \\
\leqslant & \frac{1}{p^{-}}\left[\int_{Q}|u(x)-u(y)|^{p(x, y)} K(x, y) d x d y+\int_{\Omega}|u(x)|^{\bar{p}(x)} d x\right] \\
& \quad-\quad \frac{\lambda}{r^{+}} \int_{\Omega}|u(x)|^{r(x)} d x \\
= & \frac{1}{p^{-}} \rho_{K, p(. . .)}(u)-\frac{\lambda}{r^{+}} \rho_{r(.)}(u)
\end{aligned}
$$

By Proposition 2.1 and Remark 2.1, we get

$$
\mathcal{J}_{\lambda}(u) \leqslant \frac{1}{p^{-}}\left[\|u\|_{E_{0}}^{p+}+\|u\|_{E_{0}}^{p-}\right]-\frac{\lambda}{r^{+}}\left[\|u\|_{L^{r(x)}(\Omega)}^{r+}+\|u\|_{L^{r(x)}(\Omega)}^{r-}\right]
$$

Using the Remark 2.2-(1), we obtain

$$
\begin{aligned}
\mathcal{J}_{\lambda}(u) & \leqslant \frac{1}{p^{-}}\left[\|u\|_{E_{0}}^{p+}+\|u\|_{E_{0}}^{p-}\right]-\frac{\lambda}{r^{+}}\left[\tilde{C}^{r^{+}}\|u\|_{E_{0}}^{r+}+\tilde{C}^{r^{-}}\|u\|_{E_{0}}^{r-}\right] \\
& \leqslant \frac{1}{p^{-}}\left[\|u\|_{E_{0}}^{p+}+\|u\|_{E_{0}}^{p-}\right]-\frac{\lambda}{r^{+}} \max \left\{\tilde{C}^{r^{+}}, \tilde{C}^{r^{-}}\right\}\left[\|u\|_{E_{0}}^{r+}+\|u\|_{E_{0}}^{r-}\right] \\
& \leqslant\left(\frac{1}{p^{-}}-\frac{\lambda}{r^{+}} \max \left\{\tilde{C}^{r^{+}}, \tilde{C}^{r^{-}}\right\}\right)\left[\|u\|_{E_{0}}^{p+}+\|u\|_{E_{0}}^{r+}\right]<+\infty .
\end{aligned}
$$

(ii)- Existence of the Gâteaux derivative. We define

$$
\Psi(u)=\int_{Q} \frac{1}{p(x, y)}|u(x)-u(y)|^{p(x, y)} K(x, y) d x d y, \Phi(u)=\int_{\Omega} \frac{1}{\bar{p}(x)}|u(x)|^{\bar{p}(x)} d x
$$


and

$$
\Phi_{\lambda}(u)=\lambda \int_{\Omega} \frac{1}{r(x)}|u(x)|^{r(x)} d x
$$

Then

$$
\mathcal{J}_{\lambda}(u)=\Psi(u)+\Phi(u)-\Phi_{\lambda}(u) \text { and } \mathcal{J}_{\lambda}^{\prime}(u)=\Psi^{\prime}(u)+\Phi^{\prime}(u)-\Phi_{\lambda}^{\prime}(u)
$$

- For any $u, \varphi \in E_{0}$, we have

$$
<\Psi^{\prime}(u), \varphi>=\int_{Q}|u(x)-u(y)|^{p(x, y)-2}(u(x)-u(y))(\varphi(x)-\varphi(y)) K(x, y) d x d y .
$$

Indeed,

$$
\begin{aligned}
<\Psi^{\prime}(u), \varphi> & =\lim _{t \rightarrow 0} \frac{\Psi(u+t \varphi)-\Psi(u)}{t} \\
& =\lim _{t \rightarrow 0}\left\{\int_{Q} \frac{|(u(x)+t \varphi(x))-(u(y)+t \varphi(y))|^{p(x, y)}-|u(x)-u(y)|^{p(x, y)}}{t p(x, y)} K(x, y) d x d y\right\} .
\end{aligned}
$$

Let us consider $M:[0,1] \longrightarrow \mathbb{R}$

$$
\alpha \longmapsto \frac{|(u(x)-u(y))+\alpha t(\varphi(x)-\varphi(y))|^{p(x, y)}}{t p(x, y)} K(x, y) .
$$

$M$ is continuous on $[1,0]$ and differentiable on $(0,1)$. Then by the mean value theorem, there exists $\theta \in(0,1)$ such that

$$
M^{\prime}(\alpha)(\theta)=M(1)-M(0)
$$

Then

$$
\begin{gathered}
\mid\left(u(x)-(u(y))+\left.\theta t(\varphi(x)-\varphi(y))\right|^{p(x, y)-2}[(u(x)-u(y))+t \theta(\varphi(x)-\varphi(y))](\varphi(x)-\varphi(y)) K(x, y)\right. \\
=S_{t}(u, \varphi)=\frac{|(u(x)-u(y))+t(\varphi(x)-\varphi(y))|^{p(x, y)}-|u(x)-u(y)|^{p(x, y)}}{t p(x, y)} K(x, y)
\end{gathered}
$$

Combining (3.4) and (3.5), we get,

$$
<\Psi^{\prime}(u), \varphi>=\lim _{t \rightarrow 0} \int_{Q} S_{t}(u, \varphi) d x d y .
$$

Since $t, \theta \in[0.1]$, so $t \theta \leqslant 1$, which implies

$$
S_{t}(u, \varphi) \leqslant \mid\left(u(x)-(u(y))+\left.(\varphi(x)-\varphi(y))\right|^{p(x, y)-2}[(u(x)-u(y))+(\varphi(x)-\varphi(y))](\varphi(x)-\varphi(y)) K(x, y) .\right.
$$

On the other hand

$$
S_{t}(u, \varphi) \underset{t \rightarrow 0}{\longrightarrow}|u(x)-u(y)|^{p(x, y)-2}(u(x)-u(y))(\varphi(x)-\varphi(y)) K(x, y) .
$$

Hence, by the dominated convergence theorem, we obtain (3.3).

By the same argument, we have

$$
<\Phi^{\prime}(u), \varphi>=\int_{\Omega}|u(x)|^{\bar{p}(x)-2} u(x) \varphi(x) d x \text { and }<\Phi_{\lambda}^{\prime}(u), \varphi>\lambda \int_{\Omega}|u(x)|^{r(x)-2} u(x) \varphi(x) d x .
$$

Then by relation (3.2), the result is holds. 
Continuity of the Gâteaux derivative of $\mathcal{J}_{\lambda}$. Assume that $u_{k} \longrightarrow u$ in $E_{0}$, and we will show that $\Psi^{\prime}\left(u_{k}\right) \longrightarrow \Psi^{\prime}(u)$ in $E_{0}^{*}$. Indeed,

$$
\begin{aligned}
& <\Psi^{\prime}\left(u_{k}\right)-\Psi^{\prime}(u), \varphi>= \\
& \int_{Q}\left[\left|u_{k}(x)-u_{k}(y)\right|^{p(x, y)-2}\left(u_{k}(x)-u_{k}(y)\right)-|u(x)-u(y)|^{p(x, y)-2}(u(x)-u(y))\right] K(x, y) \\
& \times(\varphi(x)-\varphi(y)) d x d y \\
& =\int_{Q}\left[\left|u_{k}(x)-u_{k}(y)\right|^{p(x, y)-2}\left(u_{k}(x)-u_{k}(y)\right) K(x, y)^{\frac{p(x, y)-1}{p(x, y)}}\right. \\
& \left.-|u(x)-u(y)|^{p(x, y)-2}(u(x)-u(y)) K(x, y)^{\frac{p(x, y)-1}{p(x, y)}}\right] \times(\varphi(x)-\varphi(y)) K(x, y)^{\frac{1}{p(x, y)}} d x d y
\end{aligned}
$$

Let us set

$$
\begin{gathered}
F_{k}(x, y)=\left|u_{k}(x)-u_{k}(y)\right|^{p(x, y)-2}\left(u_{k}(x)-u_{k}(y)\right) K(x, y)^{\frac{p(x, y)-1}{p(x, y)}} \in L^{\hat{p}(x, y)}(Q), \\
F(x, y)=|u(x)-u(y)|^{p(x, y)-2}(u(x)-u(y)) K(x, y)^{\frac{p(x, y)-1}{p(x, y)}} \in L^{\hat{p}(x, y)}(Q), \\
\bar{\varphi}(x, y)=(\varphi(x)-\varphi(y)) K(x, y)^{\frac{1}{p(x, y)}} \in L^{p(x, y)}(Q),
\end{gathered}
$$

where $\frac{1}{p(x, y)}+\frac{1}{\hat{p}(x, y)}=1$.

Hence, by the Hölder inequality (see Lemma 2.1), we obtain

$$
<\Psi^{\prime}\left(u_{k}\right)-\Psi^{\prime}(u), \varphi>\leqslant 2\left\|F_{k}-F\right\|_{L^{\hat{p}(x, y)}(Q)}\|\bar{\varphi}\|_{L^{p(x, y)}(Q)} .
$$

Thus

$$
\left\|\Psi^{\prime}\left(u_{k}\right)-\Psi^{\prime}(u)\right\|_{E_{0}^{*}} \leqslant 2\left\|F_{k}-F\right\|_{L^{\hat{p}(x, y)}(Q)} .
$$

Now, let

$$
v_{k}(x, y)=\frac{u_{k}(x)-u_{k}(y)}{|x-y|^{\frac{N}{p(x, y)}+s}} \in L^{p(x, y)}(Q) \text { and } v(x, y)=\frac{u(x)-u(y)}{|x-y|^{\frac{N}{p(x, y)}+s}} \in L^{p(x, y)}(Q) .
$$

Since $u_{k} \longrightarrow u$ in $E_{0}$. Then $v_{k} \longrightarrow v$ in $L^{p(x, y)}(Q)$.

Hence, for a subsequence of $\left(v_{k}\right)_{k \geqslant 0}$, we get

$$
v_{k}(x, y) \longrightarrow v(x, y) \text { a.e. in } Q \text { and } \exists h \in L^{p(x, y)}(Q) \text { such that }\left|v_{k}(x, y)\right| \leqslant h(x, y) \text {. }
$$

So we have

$$
F_{k}(x, y) \longrightarrow F(x, y) \text { a.e. in } Q \text { and }\left|F_{k}(x, y)\right|=\left|v_{k}(x, y)\right|^{p(x, y)-1} \leqslant|h(x, y)|^{p(x, y)-1} .
$$

Then, by the dominated convergence theorem, we deduce that

$$
F_{k} \longrightarrow F \text { in } L^{\hat{p}(x, y)}(Q) \text {. }
$$

Consequently

$$
\Psi^{\prime}\left(u_{k}\right) \longrightarrow \Psi^{\prime}(u) \text { in } E_{0}^{*}
$$

By the same argument, we show that

$$
\Phi^{\prime}\left(u_{k}\right) \longrightarrow \Phi^{\prime}(u) \text { in }\left(L^{\bar{p}(x)}(\Omega)\right)^{*} \quad \text { and } \quad \Phi_{\lambda}^{\prime}\left(u_{k}\right) \longrightarrow \Phi_{\lambda}^{\prime}(u) \text { in }\left(L^{r(x)}(\Omega)\right)^{*}
$$


Then by relation (3.2), we deduce the continuity of $\mathcal{J}_{\lambda}^{\prime}$.

The proof of Lemma 3.1 is complete.

The following result shows that the functional $\mathcal{J}_{\lambda}$ satisfies the first geometrical condition of the mountain pass theorem;

Lemma 3.2. Let $\Omega$ be a smooth bounded domain in $\mathbb{R}^{N}$ and let $s \in(0,1)$. Let $p: \mathbb{R}^{N} \times \mathbb{R}^{N} \longrightarrow(1,+\infty)$ be a continuous variable exponent with $\operatorname{sp}(x, y)<N$ for all $(x, y) \in \mathbb{R}^{N} \times \mathbb{R}^{N}$. Let (1.1) and (1.2) be satisfied. Let $r: \bar{\Omega} \longrightarrow(1,+\infty)$ be a continuous bounded variable exponent satisfy (1.7). Suppose that $K: \mathbb{R}^{N} \times \mathbb{R}^{N} \longrightarrow(0,+\infty)$ is a measurable function satisfying (1.4), (1.5) and (1.6). Then, there exists $\lambda^{*}>0$ such that for any $\lambda \in\left(0, \lambda^{*}\right)$, there exist $R, a>0$ such that $\mathcal{J}_{\lambda}(u) \geqslant a>0$ for any $u \in W_{0}$ with $\|u\|_{W_{0}}=R$.

Proof. Since $r(x)<p_{s}^{*}(x)$ for all $x \in \bar{\Omega}$, so by Remark 2.2-(1), $E_{0}$ is continuously embedded in $L^{r(x)}(\Omega)$. Then there exists a positive constant $c_{1}$ such that

$$
\|u\|_{L^{r(x)}(\Omega)} \leqslant c_{1}\|u\|_{E_{0}}, \quad \text { for all } u \in E_{0} .
$$

We fix $R \in(0,1)$ such that $R<\frac{1}{c_{1}}$. Then relation (3.6) implies

$$
\|u\|_{L^{r(x)}(\Omega)}<1 \quad \text { for all } u \in E_{0} \text { with } R=\|u\|_{E_{0}} .
$$

By Proposition 2.1-(ii), we get

$$
\int_{\Omega}|u(x)|^{r(x)} d x \leqslant\|u\|_{L^{r(x)}(\Omega)}^{r^{-}}, \quad \text { for all } u \in E_{0} \text { with } R=\|u\|_{E_{0}} .
$$

Combining (3.6) and (3.7), we get

$$
\int_{\Omega}|u(x)|^{r(x)} d x \leqslant c_{1}^{r^{-}}\|u\|_{W_{0}}^{r^{-}}, \quad \text { for all } u \in E_{0} \text { with } R=\|u\|_{E_{0}} .
$$

Using the fact that $\|u\|_{E_{0}}<1$ and (3.4), we deduce that for any $u \in E_{0}$ with $R=\|u\|_{E_{0}}$ the following inequalities hold true

$$
\begin{aligned}
\mathcal{J}_{\lambda}(u) \geqslant & \frac{1}{p^{+}} \int_{Q}|u(x)-u(y)|^{p(x, y)} K(x, y) d x d y+\frac{1}{p^{+}} \int_{\Omega}|u(x)|^{\bar{p}(x)} d x \\
& \quad-\quad \frac{\lambda}{r^{-}} \int_{\Omega}|u(x)|^{r(x)} d x \\
\geqslant & \frac{1}{p^{+}}\left[\int_{Q}|u(x)-u(y)|^{p(x, y)} K(x, y) d x d y+\int_{\Omega}|u(x)|^{\bar{p}(x)} d x\right] \\
& \quad-\quad \frac{\lambda}{r^{-}} \int_{\Omega}|u(x)|^{r(x)} d x \\
\geqslant & \frac{1}{p^{+}}\|u\|_{E_{0}}^{p^{+}}-\frac{\lambda}{r^{-}} c_{1}^{r^{-}}\|u\|_{E_{0}}^{r^{-}} \\
\geqslant & \frac{1}{p^{+}} R^{p^{+}}-\frac{\lambda}{r^{-}} c_{1}^{r^{-}} R^{r^{-}} \\
\geqslant & R^{r^{-}}\left(\frac{1}{p^{+}} R^{p^{+}-r^{-}}-\frac{\lambda}{r^{-}} c_{1}^{r^{-}}\right) .
\end{aligned}
$$

By the inequality (3.9), we can choose $\lambda^{*}$ in order to

$$
\frac{1}{p^{+}} R^{p^{+-} r^{-}}-\frac{\lambda}{r^{-}} c_{1}^{r^{-}}>0
$$


Hence, if

$$
\lambda^{*}=\frac{R^{p^{+}-r^{-}}}{2 p^{+}} \cdot \frac{r^{-}}{c_{1}^{r^{-}}},
$$

then, for any $\lambda \in\left(0, \lambda^{*}\right)$ and any $u \in W_{0}$ with $\|u\|_{W_{0}}=R$, there exists $a=\frac{R^{p^{+}}}{2 p^{+}}>0$ such that

$$
\mathcal{J}_{\lambda}(u) \geqslant a>0,
$$

which completes the proof of Lemma 3.2.

The following result shows that the functional $\mathcal{J}_{\lambda}$ satisfies the second geometrical condition of mountain pass theorem;

Lemma 3.3. Let $\Omega$ be a smooth bounded domain in $\mathbb{R}^{N}$ and let $s \in(0,1)$. Let $p: \mathbb{R}^{N} \times \mathbb{R}^{N} \longrightarrow(1,+\infty)$ be a continuous variable exponent with $\operatorname{sp}(x, y)<N$ for all $(x, y) \in \mathbb{R}^{N} \times \mathbb{R}^{N}$. Let (1.1) and (1.2) be satisfied. Let $r: \bar{\Omega} \longrightarrow(1,+\infty)$ be a continuous bounded variable exponent satisfy (1.7). Suppose that $K: \mathbb{R}^{N} \times \mathbb{R}^{N} \longrightarrow(0,+\infty)$ is a measurable function satisfying (1.3), (1.4) and (1.5). Then, there exists $\varphi \in W_{0}$ such that $\varphi \geqslant 0, \varphi \neq 0$ and $\mathcal{J}_{\lambda}(t \varphi)<0$ for any $t$ small enough.

Proof. Assumption (1.6) implies that $r^{-}<p^{-}$. Let $\varepsilon>0$ be such that $r^{-}+\varepsilon \leqslant p^{-}$. Since $r \in C(\bar{\Omega})$, then we can find an open set $\Omega_{0} \subset \Omega$ such that

$$
\left|r(x)-r^{-}\right| \leqslant \varepsilon \quad \text { for all } x \in \Omega_{0} .
$$

Consequently

$$
r(x) \leqslant r^{-}+\varepsilon \leqslant p^{-} \quad \text { for all } x \in \Omega_{0} .
$$

Let $\varphi \in C_{0}^{\infty}(\Omega)$ be such that $\bar{\Omega}_{0} \subset \operatorname{supp} \varphi, \varphi(x)=1$ for all $x \in \Omega_{0}$, and $0 \leqslant \varphi \leqslant 1$ in $\in \Omega$. Then using the above information for any $t \in(0,1)$ we have

$$
\begin{aligned}
\mathcal{J}_{\lambda}(t \varphi)= & \int_{Q} \frac{t^{p(x, y)}}{p(x, y)}|\varphi(x)-\varphi(y)|^{p(x, y)} K(x, y) d x d y+\int_{\Omega} \frac{t^{\bar{p}(x)}}{\bar{p}(x)}|\varphi(x)|^{\bar{p}(x)} d x \\
& \quad \lambda \int_{\Omega} \frac{t^{r(x)}}{r(x)}|\varphi(x)|^{r(x)} d x \\
\leqslant & \frac{t^{p-}}{p^{-}}\left[\int_{Q}|\varphi(x)-\varphi(y)|^{p(x, y)} K(x, y) d x d y+\int_{\Omega}|\varphi(x)|^{\bar{p}(x)} d x\right] \\
& \quad-\lambda \int_{\Omega_{0}} \frac{t^{r(x)}}{r(x)}|\varphi(x)|^{r(x)} d x \\
\leqslant & \frac{t^{p^{-}}}{p^{-}} \rho_{K, p(., .)}(u)-\frac{\lambda}{r^{+}} t^{r^{-}+\varepsilon} \int_{\Omega_{0}}|\varphi(x)|^{r(x)} d x \\
\leqslant & t^{r^{-}+\varepsilon}\left[\frac{\rho_{K, p(. .)}(\varphi)}{p^{-}} t^{p^{-}-r^{-}-\varepsilon}-\frac{\lambda}{r^{+}} \int_{\Omega_{0}}|\varphi(x)|^{r(x)} d x\right]
\end{aligned}
$$

Thus

$$
\mathcal{J}_{\lambda}(t \varphi)<0 \quad \text { for any } t<\xi^{\frac{1}{p^{-}-r^{-}-\varepsilon}}
$$

Where

$$
0<\xi<\min \left\{1, \frac{\frac{\lambda p^{-}}{r^{+}} \int_{\Omega_{0}}|\varphi(x)|^{r(x)} d x}{\rho_{K, p(., .)}(\varphi)}\right\}
$$

Finally, we point out that $\rho_{p(. . .)}(\varphi)>0$ (this fact implies that $\varphi \neq 0$ ).

Indeed, since $\Omega_{0} \subset \operatorname{supp} \varphi \subset \Omega$ and $0 \leqslant \varphi \leqslant 1$ in $\Omega$, so we get

$$
0<\int_{\Omega_{0}}|\varphi(x)|^{r(x)} d x \leqslant \int_{\Omega}|\varphi(x)|^{r(x)} d x \leqslant \int_{\Omega}|\varphi(x)|^{r^{-}} d x
$$


On the other hand, since $1<r^{-}<p_{s}^{*}(x)$ for all $x \in \bar{\Omega}$, then $W_{0}$ is continuously embedded in $L^{r^{-}}(\Omega)$, so there exists $c_{2}>0$ such that

$$
\|\varphi\|_{L^{r^{-}(\Omega)}} \leqslant c_{2}\|\varphi\|_{E_{0}}
$$

Combining (3.11) and (3.12), we get

$$
0<\frac{1}{c_{2}}\|\varphi\|_{L^{r^{-}}(\Omega)} \leqslant\|\varphi\|_{E_{0}}
$$

This fact and Proposition $2.1((i i)$ or $(i i i))$ imply that

$$
\rho_{p(. . .)}(\varphi)>0 .
$$

The lemma 3.3 is proved.

Our main result is given by the following theorem

Theorem 3.1. Let $\Omega$ be a smooth bounded domain in $\mathbb{R}^{N}$ and let $s \in(0,1)$. Let $p: \mathbb{R}^{N} \times \mathbb{R}^{N} \longrightarrow(1,+\infty)$ be a continuous variable exponent with $s p(x, y)<N$ for all $(x, y) \in \mathbb{R}^{N} \times \mathbb{R}^{N}$. Let (1.1) and (1.2) be satisfied. Let $r: \bar{\Omega} \longrightarrow(1,+\infty)$ be a continuous bounded variable exponent satisfy (1.7). Suppose that $K: \mathbb{R}^{N} \times \mathbb{R}^{N} \longrightarrow(0,+\infty)$ is a measurable function satisfying (1.4), (1.5) and (1.6). Then there exists $\lambda^{*}>0$ such that for all $\lambda \in\left(0, \lambda^{*}\right)$ is an eigenvalue of problem $\left(\mathcal{P}_{K}\right)$.

The proof of theorem 3.1 is based on Ekeland's variational principle and the Mountain Pass theorem.

Proof. Let $\lambda^{*}>0$ be defined as in (3.10) and let $\lambda \in\left(0, \lambda^{*}\right)$. By Lemma 3.2, it follows that

$$
\inf _{\partial B_{R}(0)} \mathcal{J}_{\lambda}>0
$$

where $\partial B_{R}(0)=\left\{u \in B_{R}(0):\|u\|_{W_{0}}=R\right\}$ and $B_{R}(0)$ is the ball centered at the origin and of radius $R$ in $E_{0}$.

On the other hand, by Lemma 3.3, there exists $\varphi \in E_{0}$ such that $\mathcal{J}_{\lambda}(t \varphi)<0$ for any $t$ small enough. Moreover, by (3.5), for all $u \in B_{R}(0)$, we get

$$
\mathcal{J}_{\lambda}(u) \geqslant \frac{1}{p^{+}}\|u\|_{E_{0}}^{p^{+}}-\frac{\lambda}{r^{-}} c_{1}^{r^{-}}\|u\|_{E_{0}}^{r^{-}} .
$$

Then we have

$$
-\infty<\bar{c}=\inf _{B_{R}(0)} \mathcal{J}_{\lambda}<0 .
$$

Combining (3.13) and (3.11), then we can assume that

$$
0<\varepsilon<\inf _{\partial B_{R}(0)} \mathcal{J}_{\lambda}-\inf _{B_{R}(0)} \mathcal{J}_{\lambda} .
$$

Applying Ekeland's variational principale to the functional $\mathcal{J}_{\lambda}: \overline{B_{R}(0)} \longrightarrow \mathbb{R}$, we find $u_{\varepsilon} \in \overline{B_{R}(0)}$ such that

$$
\left\{\begin{array}{c}
\mathcal{J}_{\lambda}\left(u_{\varepsilon}\right)<\frac{\inf }{B_{R}(0)} \mathcal{J}_{\lambda}+\varepsilon \\
\mathcal{J}_{\lambda}\left(u_{\varepsilon}\right)<\mathcal{J}_{\lambda}(u)+\varepsilon\left\|u-u_{\varepsilon}\right\|_{E_{0}}, \quad \forall u \neq u_{\varepsilon} .
\end{array}\right.
$$

So

$$
\mathcal{J}_{\lambda}\left(u_{\varepsilon}\right) \leqslant \inf _{B_{R}(0)} \mathcal{J}_{\lambda}+\varepsilon \leqslant \inf _{B_{R}(0)} \mathcal{J}_{\lambda}+\varepsilon<\inf _{\partial B_{R}(0)} \mathcal{J}_{\lambda}
$$

It follows that $u_{\varepsilon} \in B_{R}(0)$.

Now, we consider $\mathcal{I}_{\lambda}^{\varepsilon}: \overline{B_{R}(0)} \longrightarrow \mathbb{R}$

$$
u \longrightarrow \mathcal{J}_{\lambda}(u)+\varepsilon\left\|u-u_{\varepsilon}\right\|_{W_{0}} .
$$


By (3.12), we get

$$
\mathcal{I}_{\lambda}^{\varepsilon}\left(u_{\varepsilon}\right)=\mathcal{J}_{\lambda}(u)<\mathcal{I}_{\lambda}^{\varepsilon}(u) \text { for all } u \neq u_{\varepsilon} .
$$

Thus $u_{\varepsilon}$ is a minimum point of $\mathcal{I}_{\lambda}^{\varepsilon}$ on $\overline{B_{R}(0)}$. It follows that for any $t>0$ small enough and $v \in B_{R}(0)$,

$$
\frac{\mathcal{I}_{\lambda}^{\varepsilon}\left(u_{\varepsilon}+t v\right)-\mathcal{I}_{\lambda}^{\varepsilon}\left(u_{\varepsilon}\right)}{t} \geqslant 0 \text {. }
$$

By this fact, we claim that

$$
\frac{\mathcal{J}_{\lambda}\left(u_{\varepsilon}+t v\right)-\mathcal{J}_{\lambda}\left(u_{\varepsilon}\right)}{t}+\varepsilon\|v\|_{E_{0}} \geqslant 0 .
$$

When $t$ tends to $0^{+}$, we have that

$$
<\mathcal{J}_{\lambda}^{\prime}\left(u_{\varepsilon}\right), v>+\varepsilon\|v\|_{E_{0}} \geqslant 0
$$

And that gives

$$
\left\|\mathcal{J}_{\lambda}\left(u_{\varepsilon}\right)\right\|_{E_{0}^{*}} \leqslant \varepsilon
$$

From (3.17), we deduce that there exists a sequence $\left\{w_{k}\right\} \subset B_{r}(0)$ such that

$$
\mathcal{J}_{\lambda}\left(w_{k}\right) \longrightarrow \bar{c} \quad \text { and } \quad \mathcal{J}_{\lambda}^{\prime}\left(w_{k}\right) \longrightarrow 0 .
$$

From (3.14) and (3.18), we have that $\left\{w_{k}\right\}$ is bounded in $E_{0}$. Thus there exists $w \in E_{0}$ such that $w_{k} \rightarrow w$ in $E_{0}$.

By (1.7), we have that $r(x)<p_{s}^{*}(x)$ for all $x \in \bar{\Omega}$, so by Theorem 2.1 and Remark $2.2-(1)$ we deduce that $W_{0}$ is compactly embedded in $L^{r(x)}(\Omega)$, then

$$
w_{k} \longrightarrow w \text { in } L^{r(x)}(\Omega) \text {. }
$$

Using Lemma 2.1, we have

$$
\int_{\Omega}\left|w_{k}\right|^{r(x)-2} w_{k}\left(w_{k}-w\right) d x \leqslant 2\left\|w_{k}\right\|_{L^{r(x)}(\Omega)}\left\|w_{k}-w\right\|_{L^{r(x)}(\Omega)} .
$$

So, by (3.15), we get

$$
\lim _{k \longrightarrow+\infty} \int_{\Omega}\left|w_{k}\right|^{r(x)-2} w_{k}\left(w_{k}-w\right) d x=0 .
$$

Since $\bar{p}(x)<p_{s}^{*}(x)$ for all $x \in \bar{\Omega}$, by the same argument, we have

$$
\lim _{k \longrightarrow+\infty} \int_{\Omega}\left|w_{k}\right|^{\bar{p}(x)-2} w_{k}\left(w_{k}-w\right) d x=0 .
$$

On the other hand, from (3.18), we get

$$
\lim _{k \longrightarrow+\infty}<\mathcal{J}_{\lambda}^{\prime}\left(w_{k}\right), w_{k}-w>=0 .
$$

Namely

$$
\begin{aligned}
& \lim _{k}\left\{\int_{\Omega \times \Omega} \frac{\left|w_{k}(x)-w_{k}(y)\right|^{p(x, y)-2}\left(w_{k}(x)-w_{k}(y)\right)\left(\left(w_{k}(x)-w_{k}(y)\right)-(w(x)-w(y))\right)}{|x-y|^{N+s p(x, y)}} d x d y\right. \\
& \left.\quad+\int_{\Omega}\left|w_{k}(x)\right|^{\bar{p}(x)-2} w_{k}(x)\left(w_{k}(x)-w(x)\right) d x-\lambda \int_{\Omega}\left|w_{k}(x)\right|^{r(x)-2} w_{k}(x)\left(w_{k}(x)-w(x)\right) d x\right\}=0 .
\end{aligned}
$$

Hence, relations (3.20) and (3.21) yield

$$
\lim _{k \longrightarrow+\infty} \int_{\Omega \times \Omega} \frac{\left|w_{k}(x)-w_{k}(y)\right|^{p(x, y)-2}\left(w_{k}(x)-w_{k}(y)\right)\left(\left(w_{k}(x)-w_{k}(y)\right)-(w(x)-w(y))\right)}{|x-y|^{N+s p(x, y)}} d x d y=0 .
$$


Using the above information, Lemma $2.4-(i i)$, and the fact that $w_{k} \rightarrow w$ in $W_{0}$ we get

$$
\left\{\begin{array}{c}
\lim \sup <\mathcal{L}\left(w_{k}\right), w_{k}-w>\leqslant 0, \\
w_{k} \rightarrow w \text { in } W_{0}, \\
\mathcal{L} \text { is a mapping of type }\left(S_{+}\right) .
\end{array} \quad \Rightarrow w_{k} \longrightarrow w \text { in } W_{0} .\right.
$$

Then by (3.18), we obtain

$$
\mathcal{J}_{\lambda}(w)=\lim _{k \longrightarrow+\infty} \mathcal{J}_{\lambda}\left(w_{k}\right)=\bar{c}<0 \quad \text { and } \quad \mathcal{J}_{\lambda}^{\prime}(w)=0 .
$$

We conclude that $w$ is a nontrivial critical point of $\mathcal{J}_{\lambda}$. Thus $w$ is a nontrivial weak solution for problem $\left(\mathcal{P}_{s}\right)$. Finally any $\lambda \in\left(0, \lambda^{*}\right)$ is an eigenvalue of problem $\left(\mathcal{P}_{s}\right)$.

The proof of Theorem 3.1 is complete.

\section{References}

[1] E. Azroul, A. Benkirane, M. Shimi, An introduction to generalized fractional Sobolev Space with variable exponent, Preprint. https://arxiv.org/abs/1901.05687

[2] A. Bahrouni, V. Rădulescu, On a new fractional Sobolev space and applications to nonlocal variational problems with variable exponent, Discrete Contin. Dyn. Syst. 11 (2018), 379-389.

[3] G. M. Bisci, V. Rădulescu, R. Servadi, Variational methods for nonlocal fractional problems,Encyclopedia of mathematics and its applications, Cambridge University Press, Cambridge (2016).

[4] L. Caffarelli, Nonlocal diffusions, drifts and games, in nonlinear partial differential equations, Abel Symposia 7, Springer, Berlin (2012), 37-52.

[5] D.V. Cruz-Uribe, A. Fiorenza, Variable Lebesgue spaces. Foundations and harmonic analysis. Applied and Numerical Harmonic Analysis. Birkhäuser/Springer, Heidelberg, 2013.

[6] L. M. Del Pezzo and J. D. Rossi, Traces for fractional Sobolev spaces with variable exponents, Adv. Oper. Theory, 2 (2017), 435-446.

[7] E. Di Nezza, G. Palatucci and E. Valdinoci, Hitchhiker's guide to the fractional Sobolev spaces, Bull. Sci. Math, 136 (2012), no. 5, 521-573.

[8] X. L. Fan, Remarks on eigenvalue problems involving the p(x)-Laplacian, J. Math. Anal. Appl. 352 (2009) 85-98.

[9] X. L. Fan, Q. H. Zhang, Existence of solutions for $p(x)$-Laplacian Dirichlet problem, Nonlinear Analysis 52 (2003) 1843-1852.

[10] X. L. Fan, Q. Zhang and D. Zhao, Eigenvalues of $p(x)$-Laplacian Dirichlet problem, J. Math. Anal. Appl. 302 (2005), 306-317.

[11] X. L. Fan, D. Zhao, On the Spaces $L^{p(x)}(\Omega)$ and $W^{m, p(x)}(\Omega)$, J. Math. Anal. Appl, 263 (2001), 424-446.

[12] S. Goyal, K. Sreenadh Existence of multiple solutions of $p$-fractional Laplace operator with sign-changing weight function, Adv. Nonlinear Anal., 4 (2015), 37-58.

[13] U. Kaufmann, J. D. Rossi, and R. Vidal, Fractional Sobolev spaces with variable exponents and fractional $p(x)$-Laplacians, Elec. Jour. of Qual. Th, of Diff. Equa. 76 (2017), 1-10.

[14] J. Korvenpää, T. Kuusi, E. Lindgren, Equivalence of solutions to fractional p-Laplace equation, Journal de $J$. Math Pures et Applées, https://doi.org/10.1016/j.matpur.2017.10.004

[15] O. Kováčik, J. Rákosník, On Spaces $L^{p(x)}(\Omega)$ and $W^{m, p(x)}(\Omega)$, Czechoslovak Math. Jour. 41 (1991), No. 4, 592-618.

[16] N. Laskin, Fractional quantum mechanics and Lévy path integrals, Phys. Lett. A 268 (2000), 298-305.

[17] R. Metzler, J. Klafter, The restaurant at the random walk: recent developments in the description of anomalous transport by fractional dynamics, J. Phys. A 37 (2004), 161-208.

[18] M. Mihăilescu and V. D. Rădulescu, Continuous spectrum for a class of nonhomogeneous differentials operators, Manuscripta Math., 125 (2) (2008), pp. 157-167.

[19] M. Mihăilescu and V. D. Rădulescu, On a nonhomogeneous quasilinear eigenvalue problem in Sobolev spaces with variable exponent, proceedings of the american mathematical society Vol. 135, No. 9 (2007), pp. 2929-2937. 
[20] R. Servadei, E. Valdinoci, Variational methods for nonlocal operators of elliptic type, discrete and continuous dynamical systems, Volume 33, Number 5, May 2013. , pp. 2105-2137.

[21] R. Servadei, E. Valdinoci, Lewy-Stampacchia type estimates for variational inequalities driven by nonlocal operators, Rev. Mat. Iberoam., 29 (2013), no. 3, 1091-1126.

[22] R. Servadei and E. Valdinoci, Mountain Pass solutions for nonlocal elliptic operators, J. Math. Anal. Appl., 389 (2012), 887-898. 